\title{
Diacronie
}

Studi di Storia Contemporanea

$N^{\circ} 17,1 \mid 2014$

Periferie. Cultura, economia, politica

\section{La miniera e il petrolchimico}

Una questione storica nella Sardegna e nell'Italia del secondo dopoguerra

\section{Francesca Sanna}

\section{Q OpenEdition \\ 1 Journals}

\section{Edizione digitale}

URL: http://journals.openedition.org/diacronie/1063

DOI: 10.4000/diacronie.1063

ISSN: 2038-0925

\section{Editore}

Association culturelle Diacronie

Notizia bibliografica digitale

Francesca Sanna, «La miniera e il petrolchimico », Diacronie [Online], № 17, 1 | 2014, documento 7 , online dal 01 mars 2014, consultato il 10 décembre 2020. URL : http://journals.openedition.org/ diacronie/1063; DOI : https://doi.org/10.4000/diacronie.1063 


\section{Diacronie}

\section{$7 /$}

\section{La miniera e il petrolchimico \\ Una questione storica nella Sardegna e nell'Italia del secondo dopoguerra}

Francesca SANNA*

Durante il secondo dopoguerra, la Sardegna fu interessata dal declino di una delle sue più importanti attività economiche, l'estrazione mineraria. I finanziamenti pubblici erogati per la riconversione e la verticalizzazione, soprattutto coi Piani di Rinascita, si mossero però verso un modello di sviluppo polarizzato e fondato sul petrolio, comune al generale orientamento nazionale. L'esito fu la creazione di un settore industriale alieno al territorio e l'esaurimento delle miniere, nel susseguirsi di gestioni pubbliche sempre più deficitarie.

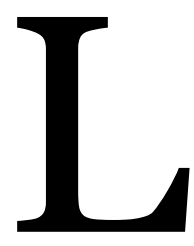

a storia della Sardegna è un mosaico dalle mille tessere, una per ogni regione che la compone. Barbagia, Sulcis-Iglesiente, Logudoro, Gallura, Ogliastra sono solo alcuni dei nomi posti a designare la geografia di una terra solcata dalla varietà: differenze economiche, sociali, culturali crearono nel passato ambienti di vita propri e peculiari, di cui anche oggi si conservano le tracce. Fra questi, il mondo minerario del Sulcis Iglesiente costituisce un unicum nella storia della Sardegna, come un tentativo di industria accolto e insieme contrastato, mai ben ambientato in una terra che risultò e risulta tutt'ora ostile a certa modificazione antropica ${ }^{1}$. L’impianto di una moderna attività industriale mineraria, a

${ }^{1}$ Il Sulcis-Iglesiente è una regione storica della Sardegna sud-occidentale caratterizzata sin dall'antichità dall'attività di estrazione mineraria ed è composta da due subregioni distinte storicamente e geologicamente: a nord l'Iglesiente metallifero e specialmente piombo-zincifero e 
partire dalla metà del XIX secolo, fu infatti caratterizzato da un continuo alternarsi di atti pionieristici e momenti stagnanti, "periodi aurei” e crisi economiche, fino al lento declino del secondo dopoguerra.

Nell'Archivio Storico Minerario di Monteponi, una delle più grandi miniere dell'isola, un affresco dipinto da Aligi Sassu nel 1950 può gettare una luce sul contraddittorio rapporto che si creò fra la miniera e il mondo circostante.

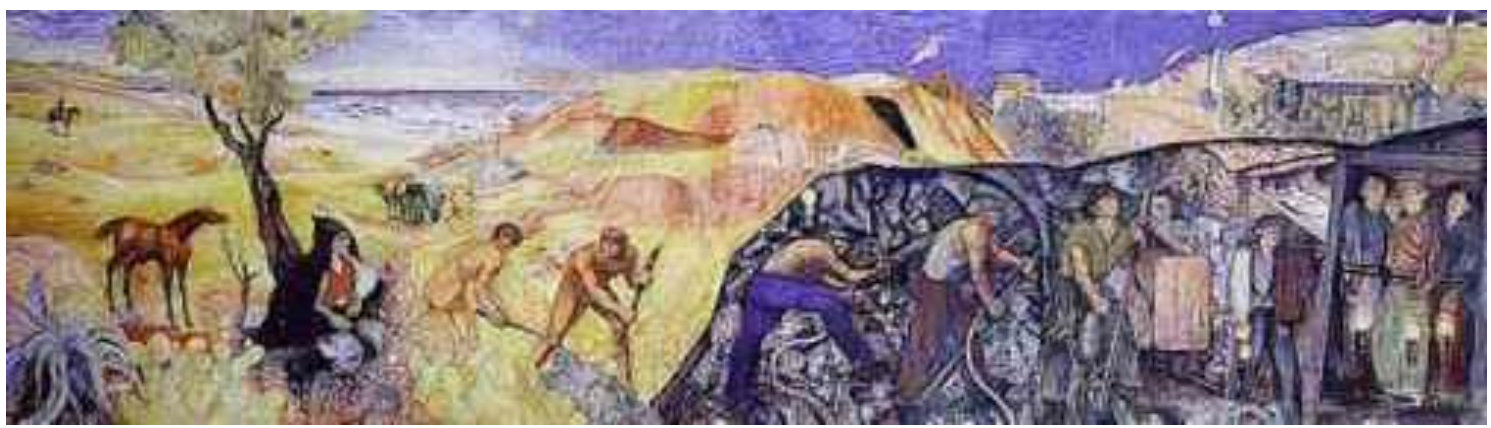

SASSU, Aligi, La Miniera, 1950, affresco, 350x1200, foresteria della miniera, Monteponi, Iglesias.

L'opera, esposta in fedele riproduzione nella sala conferenze dell'Archivio, si intitola "I tre mondi del lavoro della Sardegna" e mostra tre gruppi di uomini che incarnano le occupazioni topiche dell'isola: pastore, contadino e minatore. La raffigurazione dei personaggi non è casuale: i minatori, "uomini della notte", si trovano nell'ambiente angusto della galleria, piegati su se stessi e avvolti dal buio, mentre il contadino e il pastore, pur in una situazione di lavoro e fatica, sono dipinti in un campo aperto dalla lunga prospettiva visiva.

Il linguaggio artistico, testimone del proprio tempo, disvela qui l'ambivalenza con cui era percepito il mondo minerario in Sardegna: da una parte, accostato alle attività tradizionali, si imponeva come parte integrante della vita dell'isola, dall'altra, isolato in un ambiente chiuso, suscitava diffidenza ed estraneità in una società generalmente agro-pastorale.

Posto originariamente nella foresteria di Monteponi, l'affresco costituisce una prima chiave di lettura dell'essenza di questo mondo, in cui l'occupazione in miniera aveva risvolti non solo economici, ma anche culturali.

Nel cantiere minerario come luogo di lavoro, foriero di nuove possibilità economiche, si riversarono infatti le speranze di modernizzazione per un territorio, quello del Sulcis-Iglesiente, fino ad allora sostenuto soltanto da un settore primario arretrato e arcaico.

a sud il Sulcis carbonifero. Tav. 1. 
In questa prospettiva, la miniera diventò "fatto sociale"2 perché fu percepita come elemento integrato ai caratteri del Sulcis-Iglesiente, ad un livello che superava la sola dimensione di scambio fra salario e prestazione d'opera. Tuttavia, essa rimase oggetto di un dualismo fra celebrazione e diffidenza, così percepito a tutti i livelli della società: seppur con diverso grado di sensibilità, ognuno avvertiva lo stesso contraddittorio proporsi della "seconda questione storica della Sardegna"3.

Dopo quella agro-pastorale, la dimensione mineraria è stata appunto definita una "questione", cioè un soggetto carico di problematicità sia nella dimensione dei reali cambiamenti apportati al territorio e alla popolazione, sia nella dimensione del dibattito e della riflessione, prodotti dalla presa di coscienza dei detti cambiamenti. Per la sua lunga durata e la sua pervasività la questione è diventata anche "storica" 4 . Storico poi è anche il valore del dibattito che ritornò e ritorna tutt'ora a osservare il mondo minerario, poiché esso influenzò l'azione della politica regionale e nazionale nei riguardi della Regione a partire dalla metà del XIX e soprattutto nel XX secolo.

All'interno di questo duraturo confronto sul tema minerario, emerse il peculiare modo di percepire "l'oggetto miniera", da una parte come ricchezza, dall'altra come fonte di tensioni sociali e pericoli ambientali. Tale contrasto riverberò i suoi effetti nelle scelte di politica economica, intraprese nel secondo dopoguerra, per far fronte al lento declino dell'industria estrattiva, che incominciò a metà degli anni Sessanta. Il fatto che la miniera portasse con sé speranze di sviluppo industriale, ma che contemporaneamente mostrasse segni di decadenza (meno si pensava all'inizio ai problemi ambientali) condusse a politiche disorganiche, costellate da misure palliative come finanziamenti dispersi e indirizzati a risolvere problemi contingenti, per esempio il ripianamento dei debiti societari. Quando invece il finanziamento era mirato a sostenere interventi strutturali, come la riconversione della produzione in vista di seconde lavorazioni, esso era dissolto da scelte progettuali e tecniche legate non all'integrazione col territorio, ma al generale orientamento della politica economica

2 CICCU, Raimondo, «Prospettive dell'industria estrattiva in Sardegna», in Sardegna Economica : Quaderno 8, 11/1992, p. 48.

3 CARDIA, Maria R., Dal Piano Levi al Piano Minerario Regionale (1949-1984): 35 anni di dibattito al Consiglio Regionale, in MANCONI, Franco, Miniere e minatori della Sardegna, Cagliari, Silvana Editoriale S.p.a., 1986, p. 207.

4 La trasformazione da processo di lunga durata a problema storico è inteso qui nel modo in cui J. Le Goff lo descrive nella Prefazione a Apologia della storia di M. Bloch: «I fatti non sono dei fenomeni oggettivi esistenti senza lo storico, ma sono il risultato del lavoro e della costruzione da parte dello storico, creatore di fatti storici». LE GOFF, Jacques, Prefazione, in BLOCH, Marc, Apologia della storia o mestiere di storico, Torino, Giulio Einaudi, 1998, p. X. 
nazionale5.

Nasce qui il principio di una tendenza che giunge, fra ramificate trasformazioni, sino a tempi recenti, caratterizzati dall'emigrazione giovanile, dalla mancanza di uno sviluppo industriale integrato al territorio e dalla difficoltà di rovesciare $\mathrm{i}$ frequenti deficit di bilancio.

La storia delle esperienze di industrializzazione della Sardegna costituisce, al di là della dimensione prettamente regionale, un esempio in scala ridotta dell'orientamento della politica economica nazionale dell'epoca, focalizzato sui grandi poli industriali e sul petrolio, nel quadro del cosiddetto modello di sviluppo squilibrato.

L'isola fu infatti oggetto, dagli anni Sessanta in poi, di quella che un economista come Giulio Sapelli considera una "modernizzazione senza sviluppo", cioè una crescita relativa della potenzialità industriale, non accompagnata però da un parallelo impulso al retroterra locale, economico, sociale o culturale ${ }^{6}$.

L'esito di tale politica fu, nell'ambito minerario, quello che si può vedere oggi, anche a superficiale colpo d'occhio: lo spopolamento del territorio, dovuto all'immigrazione per sfuggire alla disoccupazione, creata dal mancato rilancio del secondo settore attraverso la verticalizzazione e la riconversione verso attività industriali legate alle risorse locali.

A ciò si aggiunge, per contro, l'ipertrofia amministrativa delle città e la presenza di poli industriali completamente alieni dal territorio, come quello di Porto Vesme, da tempo e forse da sempre oggetto di una crisi che dipende soprattutto da un assetto completamente votato all'esportazione.

Il quadro della Sardegna rispecchia inoltre un'altra tendenza peculiare degli anni del dopoguerra e del post-boom industriale e cioè, all'aggravarsi delle difficoltà economiche, il ricorso al sostegno statale e ai programmi di (speranzosi) interventi in ogni settore dell'economia.

Lungi ormai da definire questa situazione economica come il prodotto di un trattamento "coloniale" della Sardegna da parte dell'Italia (Lussu), la storia mineraria sarda non è più percepita come l'imporsi di un'élite "forestiera" sull'isola (cosa che poteva valere forse fino alla metà del secolo XX), ma come un'attività legata al settore minerario nazionale (Moro, Sabattini) e alla storia successiva dei poli petrolchimici (Ruju, Fadda), condotta verso quello scenario non dalla fuga delle società private, ma

\footnotetext{
5 Riguardo le interazioni fra politica nazionale ed investimenti nei settori industriali sardi, una puntuale ricostruzione è pubblicata in BERLINGUER, Enrico, MATTONE, Antonello (a cura di), Sardegna, Torino, Giulio Einaudi, 1998.

${ }^{6}$ Cfr. SAPELLI, Giulio, RONCHI, Veronica (a cura di), Modernizzazione senza sviluppo. Il capitalismo secondo Pasolini, Milano, Bruno Mondadori, 2005.
} 
piuttosto a causa dei caratteri dell'intervento pubblico. L'isola, dunque, si pone come uno specchio ridotto della realtà italiana, in cui dagli effetti di certe scelte (o non-scelte) si sono sviluppati fenomeni simili a quelli nazionali, ma inaspriti dal carattere isolato e originariamente difficile della Regione.

\section{1. «Una lenta ed estenuante agonia»7}

Tutta la Sardegna è interessata dal "fenomeno miniere" 8 : un carattere economico e industriale fondato sulla ricchezza naturale del suolo sardo riconosciuta già in tempi remoti, ma studiata e sistematicamente sfruttata a partire dalla seconda metà del XIX secolo.

Nel 1871 Quintino Sella, nella sua relazione sulle condizioni dell'industria mineraria in Sardegna, ne rilevava infatti l'importanza9 ${ }^{9}$, ma solo a partire dal XX secolo essa assunse caratteri industriali meno pionieristici e più razionali: meccanizzazione e nuove tecniche di estrazione e lavorazione (flottazione, elettrolisi) permisero di aumentare produttività e concentrazione del metallo nei grezzi.

Negli anni del Fascismo, la produzione fu protetta e sottoposta a regime autarchico. Il ventennio in Sardegna si caratterizzò per le vicende del bacino del Sulcis e di Carbonia, città fondata da Mussolini nel 1938 allo scopo di produrre il carbone necessario alla politica del regime e in risposta alle sanzioni comminate dalla Società delle Nazioni dopo l'invasione dell'Etiopia ${ }^{10}$.

Dopo la Seconda guerra mondiale, la Sardegna fu attraversata da contraddittorie tendenze: da un lato una situazione economica precaria e destabilizzata, dall'altro la speranza nella ricostruzione, che sembrò imprimere energia alle attività economiche.

Un periodo "aureo" per l'attività estrattiva si collocò dunque fra il 1950 e il 1960, spinto dalla domanda di materie prime e favorito anche dal basso costo della manodopera. Nel 1951 la Sardegna contribuiva, in una percentuale del 30\%, al totale della produzione mineraria nazionale e, dal censimento di quello stesso anno, si evince

\footnotetext{
7 ACCARDO, Aldo (a cura di), Politica, economia e cultura nella Sardegna autonomistica, in ACCARDO, Aldo (a cura di), L'Isola della Rinascita: cinquant'anni di autonomia della Regione Sardegna, Roma-Bari, Laterza, 1998, p. 15.

8 MEZZOLANI, Sandro, SIMONCINI, Andrea, Sardegna da salvare: paesaggi e architetture delle miniere, Nuoro, Ed. Archivio Fotografico Sardo, 1993, p. 15.

9 SELLA, Quintino, Relazione sulle condizioni dellindustria mineraria in Sardegna, Nuoro, Ilisso, 1999 [ed. orig. Relazione sulle condizioni dell'industria mineraria in Sardegna, Firenze, Tipografia Eredi Botta, 1871].

10 PERSICO, Cristina, DE ARCA, Antonella, SPADA Francesca (a cura di), Le industrie estrattive in Sardegna: analisi economica e strutturale, Cagliari, Osservatorio Economico della Sardegna, 2007, p. 11.
} 
che gli occupati attivi nel settore raggiungevano i 23.826 corrispondenti al 36,5\% degli addetti al settore secondario sardo ${ }^{11}$.

Il secondo dopoguerra, come è noto, mostrò dal punto di vista economico due facce: quella del Boom e quella degli aiuti dello stato all'economia, il cui più esteso progetto fu ovviamente la Cassa per il Mezzogiorno. Istituito il 10 agosto 1950 dal governo De Gasperi VI su proposta dell'economista Pasquale Saraceno e di altri membri del gruppo Svimez, questo ente aveva come scopo il finanziamento di attività industriali tese a migliorare la situazione economica nel Meridione ${ }^{12}$. L'azione dello Stato si sarebbe concretizzata attraverso il cosiddetto piano A.S.I., cioè un piano per la creazione di Aree di Sviluppo Industriale attraverso l'istituzione di consorzi fra Comuni, Province e Camere di Commercio, atti a imprimere energia al settore industriale e alla costruzione delle infrastrutture.

In Sardegna, i finanziamenti della Cassa furono indirizzati a trasporti, istruzione, banche, agricoltura e soprattutto a opere di bonifica delle zone acquitrinose e alla lotta antimalarica, piaghe che funestavano la Regione da tempi immemorabili. Tuttavia, nonostante gli ingenti stanziamenti, i risultati furono esigui e concentrati soprattutto sulle regioni agricole del Campidano e sui progetti dei grandi poli industriali, che si concretizzeranno poi negli anni Sessanta ${ }^{13}$. Se l'agricoltura di sussistenza diminuì radicalmente e aumentò il livello di vita dell'insieme della popolazione, l'orientamento dell'industria non si indirizzò al consumo, ma alle produzioni ad alta intensità di capitale (e bassa intensità di lavoro).

Per quanto riguarda il settore minerario, il panorama era ancora dominato dalle Compagnie private, le quali praticavano politiche individuali, votate alla quasi totale esportazione della materia grezza, impedendo così lo svilupparsi di un’industria verticalizzata di lavorazioni secondarie.

Inoltre, il secondo dopoguerra sardo si aprì in un clima di turbolenze sociali: nel

${ }^{11}$ BRIGAGLIA, Manlio, MASTINO, Attilio, ORTU, Gian G., Storia della Sardegna: La Sardegna contemporanea, vol. 5, Roma, Laterza, 2002, p. 66.

12 Sulla Cassa per il Mezzogiorno e i suoi risultati esiste una sterminata letteratura, che si è occupata non solo delle tematiche generali, di natura storica, giuridica, sociale ed economica, ma anche delle sue forme di articolazione territoriale e settoriale. Si rivela complicato proporre una sintesi della bibliografia esistente, in cui anche i pilastri fondamentali sono molti. Nonostante ciò, si propone qui un elenco che, lungi dall'avere pretese di esaustività, vuole essere una semplice indicazione per un inizio di ricerca sul tema; Cfr. PESCATORE, Gabriele, L'intervento straordinario nel Mezzogiorno d'Italia, Milano, Giuffrè, 1962; ID., «Il progetto speciale nel quadro dell'intervento straordinario», in Rivista trimestrale di diritto pubblico, 1974, pp. 885-899; SARACENO, Pasquale, «Il Mezzogiorno tra congiuntura e riforma», in Mondo economico, 38, 1972, pp. 41-46; ID., Il meridionalismo dopo la ricostruzione, Milano, Giuffrè, 1974.

${ }^{13}$ LELLI, Maurizio, Proletariato e ceti medi in Sardegna, una società dipendente, Bari, De Donato, 1975. 
febbraio-marzo del 1948 esplose infatti lo sciopero dei 72 giorni, provocato dal comportamento della SMCS (Società Mineraria Carbonifera Sarda), che violava gli accordi sui trasferimenti delle maestranze e degli impiegati. Gli operai ottennero nel dicembre un accordo con la società, ma ciò non impedì una escalation di licenziamentirappresaglia di 2.000 operai $^{44}$. Lo sciopero del 1948 dilagò poi nell'Iglesiente per 45 giorni: esso fu provocato dal tentativo delle Aziende Minerarie di destabilizzare l'unità della protesta introducendo il cosiddetto Patto Aziendale ${ }^{15}$. Contro di esso si mobilitarono 9.000 minatori in tutto il bacino metallifero dell'Iglesiente, i quali protestarono attraverso la "non collaborazione"16 o l'occupazione dei pozzi finché, nel febbraio-marzo molti, trovandosi in evidente difficoltà, tornarono al lavoro.

Le notizie di quei travagliati avvenimenti arrivarono al Consiglio Regionale attraverso l'attività dei consiglieri di opposizione socialisti e comunisti, autori di numerose interrogazioni e interpellanze ${ }^{17}$. A fronte della situazione, nell'ottobre 1952 il Consiglio istituì una Commissione Speciale per lo studio delle condizioni delle miniere sarde, ma l'Associazione Industriali insorse dichiarando l'illegittimità della Commissione, accusata di essere "presupposto al disordine" e invitando le Direzioni delle Aziende Minerarie a non rispondere alle richieste ${ }^{18}$. Il Consiglio Regionale inserì allora la commissione di studio nella Commissione parlamentare di inchiesta sulla condizione dei lavoratori in Italia, ma l'AMI (Associazione Mineraria Italiana) persistette nell'ostilità e così fecero molti Direttori, che si rifiutarono di consegnare le relazioni ai rappresentanti della Commissione Parlamentare ${ }^{19}$.

Se nel Sulcis, gli anni dal 1948 al 1957 furono caratterizzati da una stagnazione produttiva, almeno nel settore metallifero (piombo-zinco) il bilancio produttivo degli anni Cinquanta rimaneva stabilmente positivo ${ }^{20}$. Tuttavia, l'entusiasmo, generato dalla relativa prosperità, si dimostrò eccessivo e accecò lo sguardo di lungo periodo,

14 CARDIA, Maria R., op. cit., p. 207.

15 Il Patto, proposto per la prima volta dalla Direzione della Montevecchio, prevedeva aumenti salariali dal $45 \%$ al $60 \%$ previo un minimo di 20 giorni di lavoro continuato che, se disatteso per qualunque causa, avrebbe comportato la perdita di ogni diritto e del premio di collaborazione. Inoltre si imponeva la sostituzione delle Commissioni Interne ai sindacati in sede di trattativa ed era vietato il tesseramento al PSI e al PCI, cosa che palesava un intento politico. Il Patto aziendale fu poi abolito nell'aprile del 1961 in seguito ad uno sciopero e alla fusione Montevecchio-Monteponi. BERLINGUER, Enrico, MATTONE, Antonello, op.cit., p. 810.

${ }^{16} \mathrm{La}$ «non collaborazione» è un tipo di protesta attuata dai lavoratori che si recano sul posto di lavoro ma si rifiutano di attivare macchinari e svolgere i propri compiti, bloccando così la catena del ciclo produttivo. In ambito minerario la «non collaborazione» permetteva di bloccare il cantiere senza ufficialmente fare ricorso alle occupazioni, contro cui le Compagnie avrebbero reagito in modo più duro, facendo ricorso specialmente alle forze dell'ordine.

17 CARDIA, Maria R., op. cit., p. 210.

18 Ibidem.

19 Ibidem.

20 Tab. 1. 
impedendo di scorgere l'incedere di uno spettro mortale, l'impoverimento dei giacimenti, e di opporvi resistenza attraverso provvedimenti di riqualificazione o riconversione. In seguito, l'entrata dell'Italia nella CECA (1951), il crollo delle quotazioni di piombo e zinco alla Borsa di Londra (1956) e l'entrata nel MEC (1957) cancellarono le ultime compensazioni che fino a quel momento avevano tenuto a galla l'industria estrattiva sarda ${ }^{21}$.

L'entrata nella CECA ebbe ripercussioni soprattutto nel bacino del Sulcis, dove l'apertura delle frontiere commerciali eliminò la relativa protezione di cui godeva il carbone sardo.

Infatti, anche a causa della sua scarsa qualità originaria, il carbone Sulcis si vedeva preferito al carbone proveniente dall'estero, più elevato in qualità, ma anche in quantità.

Per quel che riguarda i metalli non ferrosi, il crollo della Borsa di Londra nel 1956 comportò una caduta dei prezzi di piombo e zinco, che si propagò fino al 1973 nell'ordine del $35 \%$ in media per entrambi i comparti ${ }^{22}$. Subito dopo, l'entrata dell'Italia nel MEC mise le Compagnie minerarie di fronte al problema che già aveva scosso precedentemente il Sulcis, cioè l'apertura delle barriere doganali. L'unione doganale entrò progressivamente in vigore fra il 1958 e il 1970 con l'adeguamento tariffario sulle importazioni e gli scambi fra i paesi membri e il settore estrattivo sardo ne fu penalizzato, poiché impreparato ad affrontare la concorrenza di paesi dalla produzione molto più ingente. In Italia il comparto minerario usufruì di un periodo di isolamento fra 1957 e 1966 per procedere ad un adeguamento delle strutture che avrebbe dovuto rinforzare la sua posizione commerciale, tuttavia ciò avvenne solo parzialmente o non avvenne affatto e la situazione ne fu aggravata ${ }^{23}$.

Nel 1967, vista la mancanza di «integrazione in loco della base estrattiva con quella di trasformazione» ${ }^{24}$ si decise in sede CEE di prorogare al 1969 la permanenza dell'isolamento per il comparto piombo-zincifero, che altrimenti sarebbe stato travolto dalla concorrenza. Nonostante questi accorgimenti, l'industria estrattiva sarda di soffrì

${ }^{21}$ Vedi numeri, relativi agli anni successivi a quelli indicati, del periodico curato dall'EMSa, «Industria mineraria» e CARDIA, Maria R., op. cit. per una sintesi sulla ricezione politica degli accordi europei.

${ }^{22}$ EGAM (a cura di), Piano minerario e metallurgico nazionale EGAM, Roma, EGAM, 1973, p. 77, Archivio Storico Minerario Monteponi, Iglesias (d'ora in avanti ASMM). I documenti provenienti dall'ASSM non presentano notazione archivistica in quanto l'iter di inventariazione nell'Archivio di Monteponi era ancora in essere al momento della consultazione dei documenti. 23 MINISTERO DELL'INDUSTRIA, DEL COMMERCIO E DELL'ARTIGIANATO, Relazione generale mineraria, art. 7 marzo 1973, Roma, Bardi, 1975, p. 273, ASMM.

24 EMSa, "Comunicazione dell'abbattimento dei dazi al 31 dicembre 1969», in Industria mineraria, 2/1970, p. 101. 
ripercussioni negative sia dirette (sui prezzi e quindi sui bilanci) sia indirette (sull'occupazione e sulle politiche aziendali).

I primi visibili sintomi delle difficoltà furono i licenziamenti di massa ${ }^{25}$ e le chiusure di importanti miniere come l'Argentiera nella Nurra (1962) e Ingurtosu nell'Iglesiente (1968) di proprietà della francese Pertusola, poi nel 1964 la liquidazione del settore ferrifero (miniere di Antas, San Leone e Canaglia). Il passaggio delle miniere carbonifere da SMCS a ENEL (dal 1963) costituì invece un preludio alla tendenza del decennio successivo: il trasferimento alla proprietà statale. Per fronteggiare questa nuova situazione, nel luglio 1960 il Ministero dell'Agricoltura, Industria e Commercio, accogliendo suggerimenti della CEE, invitò le società a predisporre programmi di risanamento che avessero come obiettivi il pareggio dei bilanci e in seguito il potenziamento della ricerca e la verticalizzazione ${ }^{26}$. Le società risposero elaborando programmi per il periodo 1961-1969, miranti a risolvere il problema principale, cioè la verticalizzazione, con l'obiettivo di creare un'industria integrata e competitiva a livello internazionale ${ }^{27}$. Tuttavia, presupposto indispensabile ai predetti programmi era il ripianamento dei deficit di bilancio delle società, che però non si realizzò nemmeno con la proroga dei termini di attuazione, reiterando un problema che ostacolò una potenziale riconversione del sistema al momento della chiusura dei cantieri di estrazione.

Il tipo di sfruttamento minerario condotto sino ad allora dalle Compagnie private aveva mantenuto un carattere contingente, con poca attenzione ed esigui investimenti sulla ricerca geologica di altri filoni o su nuovi metodi tecnici per ottimizzare il tipo di minerale estratto.

Perciò, nel momento in cui l'impoverimento dei giacimenti incise sempre più prepotentemente sulla produttività dei singoli cantieri, le Società non poterono avvalersi di soluzioni diverse dalla liquidazione. Per comprendere il divario fra l'invocazione alla verticalizzazione e l'effettiva attuazione di misure atte a metterla in pratica, basti pensare che all'epoca l'intero bacino minerario dell'Iglesiente contava su una sola fonderia, dislocata a San Gavino e di proprietà della Monteponi S.p.a. Le altre società, come la Pertusola, avrebbero dovuto pagare un canone per utilizzarla oppure esportare il minerale grezzo e, nella maggior parte dei casi, sceglievano quest'ultima

25 Per esempio la SMCS (Società Mineraria Carbonifera Sarda), in ordine all'attuazione di un «riassetto economico produttivo» conseguente all'applicazione delle quote CECA, licenziò 2500 operai nel 1956 e altri 1600 nel 1958. CARDIA, Maria R., op. cit., p. 212.

${ }_{26}$ MANTEGA, Paolo, Studio sui programmi di ricerche minerarie, studi e sperimentazioni realizzati e da realizzare in Sardegna, secondo le finalità dell'ART 26 della Legge $n^{\circ} 588 \mathrm{sul}$ Piano di Rinascita, Cagliari, s. e., 1973, p. 20, ASMM.

27 Ibidem. 
opzione.

Le crisi produttiva inasprì le turbolenze politiche e sociali, cominciate con la protesta del 1948. Nell'estate del 1960 si verificò un serio contrasto fra la Regione Sardegna e Pertusola, proprietaria delle miniere iglesienti di San Giovanni, Arenas, Ingurtosu, Su Zurfuru e Buggerru. In conseguenza della grave situazione di mala gestione e sfruttamento della manodopera, i sindacati avevano aperto una vertenza con l'occupazione prolungata dei pozzi per ottenere una perequazione dei salari rispetto a quelli delle miniere Pertusola ubicate in Toscana e nel Friuli Venezia Giulia (miniera di Riabl). La Pertusola assunse un atteggiamento rigido e intransigente provocando, fra maggio e luglio, scioperi sostenuti dalla CGIL e dalle camere del Lavoro di Bologna, Reggio Emilia, Milano e Torino ${ }^{28}$. Il culmine della tensione fu raggiunto in settembre, quando il Consiglio Regionale votò l'applicazione del R.D. 29.7.1927 comma 3, cioè il decreto di «non gradimento» verso il Direttore Generale della Pertusola Paul Audibert ${ }^{29}$. L'episodio segnala non solo le tendenze delle società ad una gestione «di sussistenza» delle miniere sarde, ma anche che, a livello politico, non si comprese o si ignorò la necessità di superare la sola logica di preservare senza innovare.

La realtà del declino minerario emergeva infatti sempre più esplicitamente e la reazione delle aziende fu l'abbandono delle miniere minori e la concentrazione della produzione. Dal 1961 al 1978, il settore minerario si trasformò in «campo di battaglia del pubblico contro il privato»30: le società francesi, belghe e inglesi abbandonarono i cantieri alle sigle del capitale pubblico statale (EGAM, SOGERSA, AMMI, AMMISarda, Cuprifera, ENEL), regionale (EMSa) e della multinazionale ENI (SAMIM). La politica regionale sembrò incapace di reagire alla fuga dei privati che si traduceva poi in licenziamenti di massa e liquidazione delle attività, per esempio nel caso di Ingurtosu, in cui tutti i dipendenti Pertusola furono licenziati nel passaggio a MonteponiMontevecchio. Intanto, con la Legge 588 del 1962, si erano avviati i Piani di Rinascita e, in questi, il progetto della "Supercentrale" di Porto Vesme, su cui si riversavano le speranze di creare non solo un segmento della verticalizzazione dei non-ferrosi, ma anche un bacino occupazionale alternativo al settore minerario ${ }^{31}$. Non mancarono però

\footnotetext{
28 Ibidem.
}

29 Il provvedimento consisteva nell'allontanamento del direttore e nel divieto per quest'ultimo di restare in Sardegna. Ibidem, p. 213.

30 FADDA, Paolo, Gli anni dell'Associazione degli Industriali in DEL PIANO, Lorenzo, SIRCHIA, Achille, FADDA, Paolo, Uomini e industrie. Settant'anni di storia dell'Associazione provinciale degli industriali di Cagliari nell'evoluzione dell'economia sarda, Cagliari, GAP, 1995 , p. 303.

${ }^{31}$ La legge $\mathrm{n}^{\circ} 588$ dell'11 giugno 1962 cioè il Piano di Rinascita economica e Sociale della Sardegna, con l'Art. 26 dedicato all'industria minero-metallurgica, prevedeva uno stanziamento di 11.500 milioni di lire per il periodo 1963-1974 (su 400 miliardi stanziati per l'intero Piano), il 
le disillusioni poiché, a fronte della decisione di alimentare la centrale a nafta, apparve chiaro che, con quelle scelte, inquadrate in un processo industriale basato sul petrolio, si era condannato lo sviluppo economico e sociale del Sulcis-Iglesiente ${ }^{32}$.

Gli anni Settanta si aprirono sotto i peggiori auspici per via di un contrasto fra la Regione e l'ENEL, intenzionata ad abbandonare le miniere del Sulcis. Il 22 settembre 1971 ENEL preannunciò la liquidazione di tutte le sue attività, poi procrastinata, ma intanto «volendo eludere i suoi compiti istituzionali nel settore minerario» vanificò «sistematicamente gli investimenti facendo mancare il personale che avrebbe dovuto utilizzarli e renderli produttivi» ed infine interruppe ogni attività nel luglio 197233. La condizione di oggetto passivo e non di soggetto attivo dell'attività industriale emerse proprio nel periodo della gestione pubblica, in cui la richiesta di sostegno si trasformava in attesa del salvataggio. Giunsero infatti i capitali per il risanamento societario, per il mantenimento dell'occupazione e per un'invocata verticalizzazione, ma dei tre si realizzò, per altro in modo effimero, solo la prima: l'espulsione di manodopera fu costante, la verticalizzazione sostanzialmente inesistente.

Dalla metà degli anni Settanta, la Sardegna cominciò a scrivere il capitolo finale della sua esperienza mineraria: la vita del settore fu ridotta allo stato vegetativo, l'intervento pubblico scivolò verso il salvataggio o la liquidazione delle attività ed infine si riaccese la lotta sindacale con l'occupazione dei pozzi, le vertenze e le manifestazioni

quale venne poi frazionato in 4 programmi biennali e triennali fra 1 e i 3,5 miliardi e in un piano quinquennale 1965-1969. Il Piano finalizzava questi contributi alla copertura del 50\% delle spese per impianti pilota e ammodernamenti, assumendosi anche gli oneri di ricerca. Il Piano quinquennale del 1965 prevedeva «un programma straordinario» di 6 miliardi per l'incremento della produttività nel settore minerario, a fronte dei 670 miliardi di lire per l'intero secondo settore. I motivi dell'evidente esiguità dell'intervento sono ravvisabili nelle considerazioni presenti nel Piano stesso che recita «questo settore può dare un contributo solo limitato all'espansione del settore industriale». Il Piano prevedeva inoltre la creazione dell'EMSa (Ente Minerario Sardo) per la ricerca lo studio e la sperimentazione, in modo da creare un unico centro coordinatore delle miniere destinatarie degli interventi: la gestione unitaria venne individuata come uno degli elementi basilari alla ripresa del settore, ma la reiterata presenza di questa osservazione nei documenti induce alla considerazione di una sua mancata attuazione. I piani di programmazione si configurarono come"investimento economicamente malato" sia per il loro carattere "straordinario", che li trasformò in sostitutivi e non aggiuntivi rispetto a quelli statali sia perché finalizzati, nella filosofia pubblica dell'epoca, al mantenimento dei livelli occupazionali e non a un effettivo binomio modernizzazione-integrazione che avrebbe consentito lo sviluppo. Quando alla fine degli anni ' 60 le società private abbandonarono definitivamente il campo minerario e le attività furono acquisite del capitale pubblico, la logica di gestione si indirizzò al mantenimento dei livelli occupazionali e solo in secondo luogo a obiettivi di produzione. A Porto Vesme l'AMMI aveva progettato la creazione di un impianto metallurgico Imperial Smelting, ma ancora alla fine degli anni '6o esso restava inattuato per l'opposizione dei gruppi minerari privati e per la mancata istituzione dell'EMSa, che avverrà con la Legge Regionale 8 maggio 1968 n.24, la quale avrebbe dovuto coordinare e finanziare il progetto.

${ }^{2}$ FADDA, Paolo, op. cit., p. 224.

33 CARDIA, Maria R, op. cit., p. 225. 
dei lavoratori34. Le difficoltà colpivano ancora e soprattutto le realtà più deboli: nel luglio 1977 la Commissione Industria del Consiglio Regionale fu incaricata di esaminare la situazione della popolazione del Fluminese e di Buggerru gravate dal fermo della miniera di Santa Lucia (Serramin). I lavoratori di quella miniera, collocati in cassa integrazione, non ricevevano lo stipendio da quattro mesi e, nonostante l'interessamento della Commissione, la maggior parte degli operai non si salvò dal licenziamento35. Il Consiglio Regionale continuava a rilevare la gravità della situazione, ma la lentezza dei procedimenti di unificazione gestionale, previsti dal Progetto di promozione del settore minerario e metallurgico piombo-zincifero, lo obbligarono ad attuare interventi di tamponamento tanto onerosi quanto effimeri ${ }^{36}$.

All'inizio degli anni Ottanta l'EMSa ricevette 10 miliardi di Lire per la gestione delle sue società consociate fino alla definizione delle trattative con SAMIM (di ENI) e Bariosarda (L.R. 4.9.1980 n.48), poi altri 7,3 miliardi per appianare le passività delle società confluende in SAMIM, infine fra il 1981 e il 1982, a causa di ritardi dovuti all'irrigidimento di SAMIM sulle intese sindacali, altri 5.850 milioni e 1.650 milioni per mantenersi ${ }^{37}$.

L'area dell'Iglesiente, fortemente legata alla sua storia mineraria e viziata dal carattere monoculturale, risentì enormemente del declino della produzione estrattiva, tanto che si osservò una dinamica negativa anche nei settori non direttamente legati alle miniere ${ }^{8}$. Questo fatto è imputabile alla fisionomia dell'Iglesiente come subregione economica mancante di un nucleo polarizzante, caratterizzata dal policentrismo di quella attività 39 .

Infatti, nonostante la zona fosse stata individuata come «nucleo di industrializzazione», si collocava fra le meno finanziate $(3,87 \%)$, e gli investimenti si indirizzavano al capitale e non al lavoro ${ }^{40}$. Per quanto riguarda la politica regionale, non si può dire che ci sia stata mancanza di consapevolezza della situazione, perché il problema era fra i più dibattuti: si è calcolato infatti che, nelle prime sette legislature, si

\footnotetext{
34 SABATTINI, Gianfranco, MORO, Beniamino, La crisi delle attività minerarie regionali e il ruolo del settore pubblico, Cagliari, Editrice Sarda Press, 1975, p. 85.

35 Ibidem, p. 230.

${ }^{36}$ Infatti dal 1977 tutta l'attività mineraria sarebbe dovuta passata in gestione all'ENI tramite la Società SAMIM S.p.a. Si veda OTTELLI, Luciano, Monteponi (Iglesias, Sardegna), Sassari, Delfino, 2010, p. 286.

37 CARDIA, Maria R., op cit., p. 232.

38 DETRAGIACHE, Angelo, CAMERE DI COMMERCIO, INDUSTRIA E AGRICOLTURA DELLA SARDEGNA (a cura di), Sardegna, Milano, Giuffrè, 1966, p. 88.

39 Ibidem, p. 131.

40 CLARK, Martin, La storia politica e sociale (1915-1975) in GUIDETTI Massimo (a cura di) Storia dei sardi e della Sardegna. Vol IV: L'età contemporanea, dal governo piemontese agli anni sessanta del nostro secolo, Milano, Jaka Book, 1989, p. 438.
} 
approvarono 79 O.d.G., si svolsero 526 interventi e si approvarono 13 leggi specifiche sul settore minerario ${ }^{41}$. Tuttavia, la politica regionale appare variabile in orientamento, spesso disorganica, a tratti impotente di fronte alla crisi, ora immersa nella ricerca di soluzioni ai gravissimi problemi del settore, ora dimentica della vocazione dell'Iglesiente.

Nel secondo dopoguerra, la storia dell'Iglesiente minerario e del Sulcis, a dispetto delle tante attese e temporanei successi, fu quella di una «lunga ed estenuante agonia» ${ }^{2}$ : le ultime miniere attive furono chiuse definitivamente negli anni Novanta e la gestione dei loro resti passò a società compartecipate dalla Regione Sardegna, responsabili della conservazione delle strutture abbandonate.

\section{Un destino inevitabile?}

Coloro che oggi percorrono le tortuose strade della Sardegna meridionale non possono non carpire i segni superstiti della sua passata stagione industriale: fra i monti boscosi del Sulcis-Iglesiente, appaiono i neri imbocchi di gallerie abbandonate, gli edifici fatiscenti delle laverie e delle sale argani, gli scheletri ferrosi e arrugginiti dei pozzi di estrazione. Il deserto dei cantieri fantasma, nascosti dal verde della macchia mediterranea, portano seco la suggestione del rudere, ma anche il dubbio del come o del perché lì si trovano. Molte sono le domande suscitate dai resti sparpagliati fra gli alberi: da cosa scaturì il declino minerario degli anni Sessanta? perché non si riuscì ad arrestarlo? Quale legame unisce quel passato al presente economico della Sardegna? A prima vista pare che la risposta sia semplice e cioè che il destino di una miniera si inscrive nella sua stessa natura di fonte non rinnovabile: un destino, dunque, inevitabile.

Le statistiche tendono a confermare il procedere di una crisi difficile da arrestare. Guardando alla regione metallifera dell'Iglesiente negli anni Cinquanta, la produzione di piombo e zinco mantenne livelli stabili con un minimo decremento del 2,04\% dal 1951 (226.804 tonn) al 1961 (222.168 tonn)43 coprendo il 67\% della produzione italiana al 196044. In seguito, dal 1960 al 1972, si assistette ad un crollo che sfiorava il 50\% per un comparto che nel 1972 costituiva il 56\% del settore estrattivo sardo e il 53,77\% del

\footnotetext{
${ }^{41}$ ACCARDO, Aldo, op. cit., p. 15.

42 Ibidem.

43 DETRAGIACHE, Angelo, op. cit., p. 66.

44 COLLEGIO NAZIONALE SINDACATO INGEGNERI DEL MINISTERO PER L'INDUSTRIA E IL COMMERCIO (a cura di), Centenario del Corpo delle Miniere, 186o-196o, Faenza, Stabilimento grafico Lega, 1960, p. 184.
} 
valore prodotto su tutta la produzione mineraria regionale45. Alla diminuzione della produzione si accompagnò una parallela concentrazione degli organici: nel 1951 il settore piombozincifero occupava quasi 9.000 lavoratori (circa la metà di tutto il settore estrattivo sardo), mentre nel 1961 si scese a circa 5.00o e all'inizio degli anni Settanta se ne contarono poco più di 3.00046. Durante il decennio 1970-1980 l'occupazione subì un ulteriore decremento di più di 1000 lavoratori scendendo a quota 1.846 unità nel 197947.

Il percorso discendente di tutti i valori induce a considerare il trentennio 1950-1980 come un periodo di progressivo declino del comparto, nonostante si possano cogliere, nei documenti delle società minerarie, fiduciose attribuzioni di ruolo trainante o di impulso all'economia isolana.

La forza lavoro si riversò allora nell'edilizia o nei flussi migratori verso il "Continente", che provocarono un cospicuo decremento della popolazione pari a quasi un quarto degli abitanti dell'Iglesiente fra 1951 e 196148. A fronte di questi dati, il destino delle miniere appare a posteriori già segnato, tuttavia il determinismo è sempre una risposta liquidatoria, perché ogni attività economica, seppur viziata da problemi strutturali, nasce, si sviluppa e termina secondo il modo in cui la si conduce e procede secondo le scelte degli attori coinvolti.

Nelle miniere sarde l'attività industriale fu spesso ostacolata da problemi strutturali, "caratteri oggettivi dell'attività», come la dispersione naturale e la dimensione dei giacimenti, la qualità e il tenore dell'estratto, lo stato delle riserve e l'incidenza dello sfruttamento ${ }^{49}$.

La dispersione dei giacimenti aveva da tempo indotto una localizzazione a carattere puntiforme dei cantieri la quale, assommandosi alle carenze infrastrutturali e alla difficile morfologia del territorio, aggravò ulteriormente i costi di trasporto e la questione dell'integrazione produttiva. Questa geografia industriale fu affiancata e, per certi versi, dipese dalla dimensione medio-piccola o esigua dei giacimenti che, conseguentemente, comportarono sia uno scarso tonnellaggio produttivo sia l'impossibilità di impiegare tecnologia di grande scala ${ }^{5}$. Dal punto di vista mineralurgico, nel secondo dopoguerra i giacimenti sardi si presentavano impoveriti nel tenore e nella quantità di estratto, inquinato da altri componenti: zolfo nel carbone

45 SABATTINI, Gianfranco, MORO, Beniamino, op. cit., pp. 28-29.

46 Ibidem cit., p 14.

47 Tab. 2 e Tab. 3, Cfr. Notiziario tecnico-economico EMSa, 1-2/1980, p. 61.

48 DETRAGIACHE, Angelo, CAMERE DI COMMERCIO, INDUSTRIA E AGRICOLTURA

DELLA SARDEGNA (a cura di), op. cit., p. 157.

49 SABATTINI, Gianfranco, MORO, Beniamino, op. cit., p. 67.

${ }^{5}$ DETRAGIACHE, Angelo, op. cit., p. 12. 
Sulcis, zinco nel ferro, argille e silice nel piombo e nello zinco5노. A ciò si deve aggiungere l'irregolarità e la profondità delle mineralizzazioni che condusse ad un sempre maggior approfondimento dei pozzi di coltivazione.

Le escavazioni profonde non solo opposero un maggior costo per le difficoltà di lavoro in sotterraneo (acqua da pompare, ventilazione e sostegno della galleria), ma soffrirono anche dell'ulteriore abbassamento dei tenori, effetto naturale dei processi geologici di mineralizzazione ${ }^{52}$. Infine, nella povertà quantitativa dei giacimenti si può individuare un processo di esaurimento di ordine storico: il «vizio di origine» delle aziende del settore, cioè la monocoltura mineraria, si ricollega al continuo e reiterato rilascio di concessioni sulle medesime zone le quali, in forza del carattere naturalmente esauribile della risorsa minerale, si sono progressivamente impoverite53. Tuttavia, ancora più determinanti per l'aggravarsi della crisi furono le scelte, ma soprattutto le non scelte, degli attori economici.

\section{Verticalizzazione: questo è il problema?}

Già nel 1966, come sottolineato dallo studio delle Camere di Commercio, Industria e Agricoltura, vi era la consapevolezza del potenziale pericolo dovuto alla mancanza di verticalizzazione:

Si è parlato tanto di verticalizzazione: una condizione essenziale perchè l'industria mineraria sarda facesse un salto di qualità, passando da una fase di pura e semplice produzione e commercio dei mercantili alla produzione dai suoi minerali di beni a più alto valore aggiunto54.

La produzione mineraria è costituita da tre fasi - estrazione, lavorazione,

${ }^{51}$ La presenza dello zolfo nel carbone Sulcis è individuata fra il 3 e il $5 \%$, valore definito notevole, in CAVINATO, Antonio, Depositi minerari: con speciale riguardo alle miniere ed ai materiali utili italiani, Torino, Libreria Tecnica V. Giorgio, 1952, p. 115. « [...] uno dei motivi che ne rendono difficile l'uso se non in seguito ad arricchimento». Ibidem, p. 233. Nella nota di Mario Steri, direttore dei lavori della laveria Mameli di Monteponi, si afferma: «purtroppo le speranze di ottenere concentrati di buona qualità si realizzarono solo parzialmente per la presenza di silice solubile nei prodotti» in STERI, Mario, Le laverie delle miniere di Monteponi, anni 1960-1965, Iglesias, CTE Iglesias, 2005, cit. in OTTELLI, Luciano, op. cit., p. 275.

${ }^{2}$ ZUFFARDI, Piero, Le risorse minerarie in Sardegna, in I problemi storici della Sardegna : atti del Convegno di studi tenuto a Pavia il 12-13 ottobre 1985, Milano, Cisalpino-Goliardica, 1986, p. 63.

53 S.a., «Programma generale straordinario di ricerca: metodologie e finalità» in Notiziario tecnico-economico EMSa, 3/1973, p. 7.

54 SALVATORI, Ilio, ZUFFARDI, Piero, I giacimenti minerari in MANCONI, Francesco (a cura di), op. cit., p. 142. 
raffinazione - che costituiscono una unità processuale, ma possono essere svolte o da imprese separate tra loro o da un'unica impresa, attraverso il cosiddetto processo di verticalizzazione dell'industria estrattiva. La scelta fra separazione o concentrazione dipende da molteplici fattori di natura tecnica (caratteri naturali delle miniere che comportano determinate scelte di tecnologia applicabile), economica e politica55. L'applicazione del processo di verticalizzazione a gruppi di società comporta da un lato la creazione di economie di scala con l'eliminazione di costi comuni, dall'altro irrigidisce la gestione perchè organizza gli impianti secondo uno schema dato.

In Sardegna, molte volte fu posto l'obiettivo di verticalizzare, ma sia la gestione privata sia quella pubblica lo mancarono, definendo così una frattura non sanata fra le fasi produttive ${ }^{5}$.

Per il comparto piombo-zincifero, il processo di verticalizzazione seguì un percorso travagliato, dibattendosi fra la monocoltura e la mancanza di una struttura industriale differenziata, finché alla fine degli anni Cinquanta si intraprese una strada, quella degli impianti di elettrolisi di zinco di Porto Vesme, che però ripropose una situazione di estraneità rispetto al territorio. Nel pieno degli anni Settanta, quando ormai la crisi era inarrestabile, la politica gestionale dell'EGAM riprese ancora il problema in termini più di previsione che di effettiva attuazione, in cui mancava del tutto la prospettiva di un'inversione verso impianti di seconda lavorazione, cioè la raffinazione, del processo produttivo minerario. Si era consapevoli che «le prospettive dipendevano dalla capacità di inserirsi nella tendenza a mettere in moto processi di verticalizzazione per creare condizioni strutturali-produttive che avrebbero superato situazioni di "scambio ineguale" con l'estero, liberandosi da strozzature e subordinazioni che stavano alla base della crisi»57, ma anche i grandiosi progetti per il polo di Porto Vesme comportarono conseguenze di sviluppo fittizio, figlio di un modello di industrializzazione squilibrato. Il Consiglio Regionale, a margine della "Conferenza nazionale Mineraria di Cagliari", ribadiva:

l'interesse della Sardegna [...] all'unificazione delle aziende piombozincifere e al loro collegamento con un sistema di trasformazione dei metalli non ferrosi che realizzasse nell'isola un'industria metallurgica di livello

\footnotetext{
55 Ibidem, p. 257.

56 SABATTINI, Gianfranco, MORO, Beniamino, op. cit., p. 90.

57 A.MI.ME, Associazione Minatori Memoria (a cura di), Atti del Convegno delle Regioni minerarie "Miniere, Regioni, Programmazione Nazionale" Firenze 6-7-8 luglio 1973 in Sardegna: minatori e memorie, Cagliari, Arti Grafiche Pisano Srl, 2006, p. 112.
} 
nazionale e mediterraneo ${ }^{58}$.

Infatti, nel 1974 finalizzò il rifinanziamento del Piano di Rinascita alla «creazione e sviluppo, mediante le necessarie verticalizzazioni e unificazioni [...] di una moderna base di trasformazione mineralurgica, metallurgica, manifatturiera di minerali non ferrosi» salvo poi doversi confrontare con i bilanci deficitari delle società di gestione deputate a realizzare questo progetto59. Il modello pensato con i Piani di Rinascita dagli anni '6o si basava sulla creazione di poli di sviluppo con al centro grandi industrie di base, che avrebbero trainato e integrato la nuova struttura industriale in un sistema economico di raggio nazionale e internazionale ${ }^{60}$. Il modello di industrializzazione per poli venne però intrapreso da una Regione evidentemente sottosviluppata, per la quale non era possibile applicare le stesse logiche di altre realtà dal tessuto economico già vivace, perciò il modello assunse connotati marcatamente polarizzati e soprattutto scollegati dalla media industria regionale di trasformazione.

Infatti, lo stabilirsi dell'industria chimica in Sardegna fu un calarsi dall'alto favorito dall'orientamento del Piano di Rinascita, che prevedeva e finanziava una trasformazione strutturale nel senso di una industrializzazione di base a bassa intensità di manodopera ${ }^{61}$. Ispirati a un modello di gigantismo, questi poli industriali assunsero ben presto i connotati di cattedrali nel deserto ${ }^{62}$ e disgregarono i già precari equilibri fra la produzione e mercato sardo. Non potendo assorbire prodotti industriali di livello avanzato, il sistema assunse evidenti connotati di esogeneità con una marcata tendenza all'esportazione ${ }^{63}$. La crisi del settore minerario sardo si può allora mettere in relazione a questo modello poichè, scollegato dal mercato interno che non inviava impulsi per la mancanza di imprese di seconda lavorazione, esso si rivolse all'esterno, dove però non raggiunse il grado di competitività necessaria a sopravvivere al confronto con altre

${ }^{8}$ CARDIA, Maria R., op. cit., p. 228.

59 Ibidem.

6o BOSCOLO, Alberto, BULFERETTI, Luigi, DEL PIANO, Lorenzo, SABATTINI, Gianfranco, Profilo storico economico della Sardegna dal riformismo settecentesco ai piani di rinascita, Milano, Franco Angeli, 1991, p. 199.

${ }^{61}$ SAPELLI, Giulio, op. cit., p. 169.

${ }_{62}$ L'espressione indica la costruzione di impianti industriali caratterizzati da gigantismo e assenza di integrazione alle attività economiche del territorio. Paradigmatico è, nel caso sardo,l'esempio dell'industria petrolchimica di Ottana costruita nel 1973, trattato in BERLINGUER, Enrico, MATTONE, Antonello, op. cit.

63 «La strategia dello sviluppo alla base del Piano quinquennale della Sardegna può essere configurata come un'azione volta a modificare la struttura dell'offerta regionale, non per adeguarla alle esigenze quantitative e qualitative del mercato isolano, ma per consentirle di inserirsi nel mercato nazionale ed estero" in REGIONE AUTONOME DELLA SARDEGNA Piano di rinascita economica e sociale delle Sardegna (L.11.6.1962, N. 588 e L.R. 11.7.1962 N. 7.): piano quinquennale 1965-1969, Cagliari, Regione Autonoma della Sardegna, 1966, p. 26. 
realtà minerarie.

La strategia adottata dalle società per ovviare ai problemi dell'industria estrattiva fu allora quella della programmazione: proiezioni, previsioni, programmi, piani di ricerca costituiscono una gran parte dei documenti presenti negli archivi degli enti che si occupano di conservare la storia industriale della Sardegna. Tuttavia, nonostante l'affermazione della volontà di pianificare (almeno dagli anni Sessanta), non si mise in pratica un'effettiva organizzazione razionale del settore: si scrissero e si dichiararono obiettivi di produzione o di unità gestionale ${ }^{64}$, ma la loro attuazione fu ben lungi dall'inverarsi. Il capitale privato, soprattutto dalla fine degli anni Cinquanta, tralasciò le fase di ricerca e valutazione giacimentologica per gettarsi esclusivamente sullo sfruttamento del minerale sperando di appianare così i propri deficitari bilanci ${ }^{65}$. In tal modo vennero progressivamente esaurendosi non solo le riserve materiali, ma anche le conoscenze tecnico-scientifiche necessarie per ovviare a quel declino. Dopo un'indagine fra il 1967 e il 1969, così scrisse la Commissione Consiliare per l'industria

come si vede, attualmente il disordine governa l'attività mineraria in Sardegna. Gli imprenditori hanno sempre operato e continuano ad operare ciascuno per proprio conto. L’indagine ha offerto alla Commissione un quadro del tutto frammentario e disorganico ${ }^{66}$.

Negli anni Settanta l'intervento del pubblico, pur creando svariate società di gestione e ricerca (EGAM, EMSa, SOGERSA...), non migliorò il quadro organizzativo, ancora una volta orfano di una visione d'insieme «strategicamente rivitalizzante» dell'intero comparto ${ }^{67}$. Alla fine degli anni Settanta, la Nota di specificazione operativa del progetto minerario, metallurgico e manifatturiero, ribadendo lo stato di crisi del settore estrattivo, evidenziò una «politica gestionale di sopravvivenza, caratterizzata dal contenimento massimo delle spese» ${ }^{68}$. La sfida industriale era stata affrontata con piani produttivi generici e poco adeguati conducendo alla polverizzazione degli interventi. Le previsioni furono sistematicamente sconfessate dai rendiconti di fine anno e i piani programmatici non trovarono mai piena realizzazione: da una situazione tale discese la dispersione delle energie e dei finanziamenti ${ }^{69}$.

64 EGAM (a cura di), op. cit., pp. 14 e 83, ASMM e EMSa (a cura di), op. cit., p. 17, ASMM.

65 SALVATORI, Ilio, ZUFFARDI, Paolo, op. cit., pag 142.

66 CARDIA, Maria R., op. cit., p. 220.

${ }_{67}$ FADDA, Paolo, op. cit., p. 344.

68 CARDIA, Maria R., op. cit., p. 230.

69 Confrontando i già citati Piani Programmatici di EMSa, EGAM e Piombozincifera Sarda degli anni Settanta con i Notiziari EMSa degli anni Settanta e Ottanta si evince l'esistenza di una 
La verticalizzazione era una questione di primordine soprattutto in relazione alla situazione internazionale. Dagli anni Sessanta, giunse poi a compimento una decisa traslazione dei centri decisionali dei prezzi dei minerali nei continenti extra-europei, da Londra all'altra sponda del'Atlantico: il settore minerario italiano si configurò allora come area a «sovranità economica limitata» $7^{0}$, maggiormente sottoposta alla fluttuazione del corso internazionale dei prezzi. Nel quadro critico delineatosi dopo lo shock del 1973, i gruppi oligopolistici americani e europei si impegnarono in uno scontro in cui i primi emersero con successo: tendendo a verticalizzare solo nelle proprie industrie metropolitane, essi dominavano il mercato da una posizione ben munita, accumulando rendita nell'headquarter e riducendo al minimo le dispersioni finanziarie nei paesi di estrazione ${ }^{71}$. Questa politica schiacciò le imprese meno propense all'innovazione, come quelle della sarde, in quel momento gestite con logiche del salvataggio dal capitale pubblico ${ }^{72}$. Le imprese con un esiguo numero di miniere non verticalizzate subirono le variazioni dei prezzi senza poterli influenzare, essendo marcata la predilezione del mercato verso i trasformatori piuttosto che verso i produttori di materie prime.

L'Italia e la Sardegna, si trovarono in quel momento ad affrontare anche l'unione doganale europea, in conseguenza della quale si prospettò un adeguamento delle strutture, in primis lo sviluppo delle lavorazioni secondarie. Per agevolare l'operazione il comparto minerario italiano usufruì di un periodo di isolamento fra 1957 e 196673, tuttavia la verticalizzazione avvenne solo parzialmente o non avvenne affatto, così nel 1967, in sede CEE, fu prorogato per evitare che il settore minerario italiano fosse travolto, «mancando di integrazione in loco della base estrattiva con quella di trasformazione»74. In previsione dell'avvio del nuovo mercato, invece di modernizzare le strutture, le società minerarie in Sardegna assunsero provvedimenti che scaricarono i costi del cambiamento sui lavoratori: la Società Monteponi, la Società Montevecchio, AMMI, Pertusola e Ferromin avviarono dalla seconda metà del 1957 licenziamenti e riduzioni dell'orario di lavoro per preservare l'equilibrio finanziario ${ }^{75}$. Dunque le società non agirono nel senso di un rinnovamento strutturale di lungo periodo, ma operarono

discrepanza fra le proiezioni degli esiti d'investimento, riguardanti soprattutto mantenimento dell'occupazione e ripianamento dei debiti, e gli esiti stessi.

$7^{\circ}$ ROSSI, Giovanni, «Considerazioni sulla situazione mineraria in Sardegna» in Sardegna Economica : quaderno 8, Cagliari, 11/1992, p. 40.

${ }^{71}$ Conferenza Nazionale Mineraria. Cagliari 9/11 marzo 1973. Atti cit., p. 348.

${ }^{72}$ CICCU, Raimondo, op. cit., p. 48.

73 MINISTERO DELL'INDUSTRIA, DEL COMMERCIO E DELL'ARTIGIANATO (a cura di), $o p$. cit, p. 273.

74 CARDIA, Maria R., op. cit., p. 220.

75 Ibidem, p. 214. 
cambiamenti contingenti per ovviare temporaneamente al deficit di bilancio. A fronte di tale comportamento, il Consiglio Regionale approvò un O.d.G. (O.d.G $\mathrm{n}^{\circ} 4$ 10.10.1957) che impegnava la Giunta a intervenire per bloccare un flusso dei licenziamenti, quantificabile in circa 4.000 unità nell'intero settore, di cui circa 2.000 solo nel comparto piombo-zincifero ${ }^{76}$. Provvedimenti di tal fatta, volti a salvare le attività agendo esclusivamente sul costo della manodopera, non poterono non provocare numerose agitazioni sindacali e sociali: nell'estate calda del 1960 molte miniere, fra cui quelle della Pertusola nell'Iglesiente, furono teatro di vertenze e occupazioni dei pozzi che proseguirono fino a metà del 1961.

La Sardegna mineraria si trovò dunque nel secondo dopoguerra catapultata in un circuito internazionale popolato da concorrenti aggressivi e superiori per potenzialità produttiva e livello tecnologico fra i quali, impreparata, si perse in un'area marginale della competitività. Tuttavia, il declino non fu un improvviso lampo precipitato come fatale destino sull'isola, poiché già negli anni Cinquanta, periodo di relativo benessere, un'oculata osservazione poteva scorgere i primi segni di squilibrio. A tal proposito, è la fuga delle multinazionali private come la Pertusola a segnalare l'intuito pericolo di scivolamento nella crisi: le società abbandonarono il campo prima di restarne intrappolate. Certo, è a posteriori che si connette il processo con l'evento, ma all'epoca i privati avvertirono prima del pubblico la china in cui l'isola stava pericolosamente scivolando e che non poteva essere affrontata con la vigente struttura economica non verticalizzata. Il pericolo maggiore, cioè l'esaurimento delle risorse, era emerso prima tenue, con le diseconomie fra costi di produzione e rendimento, e poi sempre più oneroso, nonostante i seppur minimi miglioramenti tecnologici. Le società private captarono l'avvertimento e abbandonarono la zavorra al pubblico che la caricò sulle proprie spalle finché piani di rilancio sempre più utopici non resero evidente l'assurdità di continuare a considerare il settore come attiva ricchezza produttiva.

La storia mineraria della Sardegna si costruisce a partire da un'esperienza industriale complessa e a tratti anche contraddittoria, perché vede intrecciati segni di profondo radicamento e insieme estranea coesistenza col territorio. La vita della Sardegna e delle sue miniere può infatti apparire da un lato convergenza organica, per le modificazioni materiali e culturali che si realizzarono nel corso del tempo, ma dall'altro uno scontro mai sopito, in cui la dinamica azione-reazione fra i protagonisti comportò una situazione di crescita senza sviluppo.

La Sardegna conserva però molteplici “eredità minerarie”, fra cui il lascito materiale

${ }^{76}$ OPPO, Anna, La vita in miniera negli anni '5o, in MANCONI, Francesco (a cura di), op. cit., p. 191. 
della miniera: le costruzioni, le discariche di materiale inerte o le montagne traforate dalle gallerie non possono che testimoniarlo, ma il loro stato di abbandono è indice della subalternità dell'isola all'applicazione delle tecniche estrattive al proprio corpo fisico.

Tuttavia, l'attività estrattiva provocò anche un processo graduale di cambiamento della società, che dal punto di vista culturale si presenta come onda lunga, tendenza estesa attraverso i tempi. Da una parte essa emerge, materiale e concreta, attraverso l'ibridazione dei modelli urbani del Sulcis Iglesiente, dall'altra interessa la storia della vita quotidiana, investita da un mutamento radicale nella dimensione del lavoro e della percezione del tempo.

Nel caso dell'urbanizzazione del Sulcis Iglesiente, la miniera ha sovrapposto elementi nuovi a borghi essenzialmente agro-pastorali, come costruzioni legate all'attività mineraria (magazzini, pozzi di estrazione, laverie), ma anche opere infrastrutturali (strade, ferrovie, canalizzazioni idriche) e nuovi palazzi ospitanti case operaie, ospedali, scuole e anche strutture sportive.

Pur conservando la loro originaria organizzazione (case generalmente basse e "familiari", strade strette e tortuose, una o poche vie principali di collegamento con altri paesi) il loro modello di urbanizzazione è riconoscibile come «habitat minerario»77, anche se il caso propone caratteri peculiari.

Studiato dal professor Pasquale Mistretta, Rettore dell'Università di Cagliari, l'habitat mineraro dell'Iglesiente è ibrido, perché giunto a modificare uno stato di urbanizzazione in parte già esistente, in primis ampliandolo in dimensioni, grazie all'aumento demografico generato dal miglioramento del reddito familiare degli abitanti, e poi anche con le costruzioni citate più sopra.

L'influsso della miniera fu però recepito in modi diversi: in alcuni casi rimase temperato dal pre-esistente carattere agro-pastorale, in altri si legò indissolubilmente al destino dell'attività estrattiva, tanto da condurre, dagli anni della crisi mineraria, prima a un graduale spopolamento e poi al completo abbandono di centri come il villaggio di Arenas e il paesino di Ingurtosu.

Il punto discriminante fu appunto il diverso grado di dipendenza dall'attività estrattiva, che, non superando mai la monocolturalità, non propose alcuna alternativa economica post-mineraria alla popolazione di quei borghi sviluppatisi da originari

77 Il concetto di habitat designa ambienti di sviluppo umano, caratterizzati da un certo tipo di utilizzazione delle risorse, la quale crea peculiari strutture incidenti economicamente, socialmente e culturalmente sulla zona interessata, in modo duraturo e pervasivo. Per approfondire il tema si veda: MISTRETTA, Pasquale, Gli habitat minerari della Sardegna in MANCONI, Francesco, op. cit., pp. 115-139. 
villaggi operai sorti a ridosso dei cantieri.

Il carattere di doppia natura dei paesi che invece sopravvissero, seppur con difficoltà, si ritrova nelle considerazioni sul sistema di vita, sulle abitudini e su tutto ciò che concerne la "cultura del quotidiano" degli abitanti del luogo.

Da sempre abituati ad un ritmo "agricolo" dell'esistenza, ritmo di "attesa", legato alla natura, alle stagioni, alle pause necessarie alle colture, gli abitanti che entrarono a lavorare in miniera si ritrovarono a far fronte a un modo di concepire il lavoro completamente estraneo e incomprensibile, per lo più imposto dall'alto.

La nuova e rigorosa disciplina della miniera, l'irregimentazione della manodopera, il tentativo di creare gruppi di lavoro coesi e fidelizzati, attraverso istituzioni di dopolavoro e villaggi operai, incisero fortemente, ma non ribaltarono del tutto il modello di vita dell'area, ridefinendo perciò un sistema ibrido di «vita a due tempi» 78 .

La resistenza al cambiamento e una concezione temporanea del lavoro in miniera come parentesi nella vita del contadino, del pastore o anche dell'artigiano, necessaria a guadagnare un piccolo capitale da utilizzare nella propria attività principale - da un lato fece fallire il tentativo delle società di creare paternalisticamente una propria classe operaia, dall'altro sconvolse i ritmi degli abitanti, che cercarono di adattarsi senza però perdere il loro modus vivendi originario.

In un alternarsi fra resistenza e accettazione della scansione oraria dell'industria, la "vita a due tempi" rappresentava la divisione della giornata del lavoratore fra casa e miniera, percepiti come due mondi diversi, regolati da tempi diversi appunto.

La separazione fra casa e luogo di lavoro era quindi definita dal percezione del tempo: tempo tradizionale e tempo nuovo, quest'ultimo sovente avvertito come nemico e straniero, tanto più che i ritmi di lavoro furono, almeno fino alla famosa "cacciata" dell'Ing. Audibert, regolati dal cronometro e dal cottimo.

L'esito del compromesso fra casa e lavoro fu allora un comportamento di amoreodio verso la miniera, che da un lato speranza di maggior guadagno e dall'altro estranea al proprio modo di vivere.

Nonostante ciò, la miniera penetrò, come oggetto fondamentale alla definizione della sub-regione Sulcitana e Iglesiente, nel processo culturale e nella coscienza identitaria locale, visibile dall'esterno - ed esplicitamente - anche solo attraverso i molteplici murales a tema minerario che adornano i muri delle case di questi paesi.

L'importanza regionale dello studio del passato estrattivo della Sardegna, è ribadito

${ }^{78}$ Per approfondire il tema della visione del mondo dei sardi del Sulcis Iglesiente si rinvia ad OPPO, Anna, op. cit. pp. 191-198; ATZENI, Paola, «Lavoro e Tempo in miniera» in La Ricerca Folklorica, 10/2008, pp. 121-136. 
poi dalle recenti dinamiche economiche dell'isola79, ancora legate alle scelte nazionali degli anni del dopoguerra, che inducono ad approfondire le dinamiche del mondo minerario, rivelatrici di quel passato senza il quale saremmo vinti dall'ignoranza del presente.

${ }^{79} \mathrm{Si}$ fa riferimento alle vicende dello stabilimento ALCOA di Porto Vesme e della società Igea S.p.a., titolare della gestione dei cantieri minerari, della bonifica del territorio e dell'organizzazione dell'Archivio Storico Minerario di Monteponi. 


\section{Appendice}

\section{Tavola 1 - la Sardegna mineraria, confronto concessioni 1921-1973}
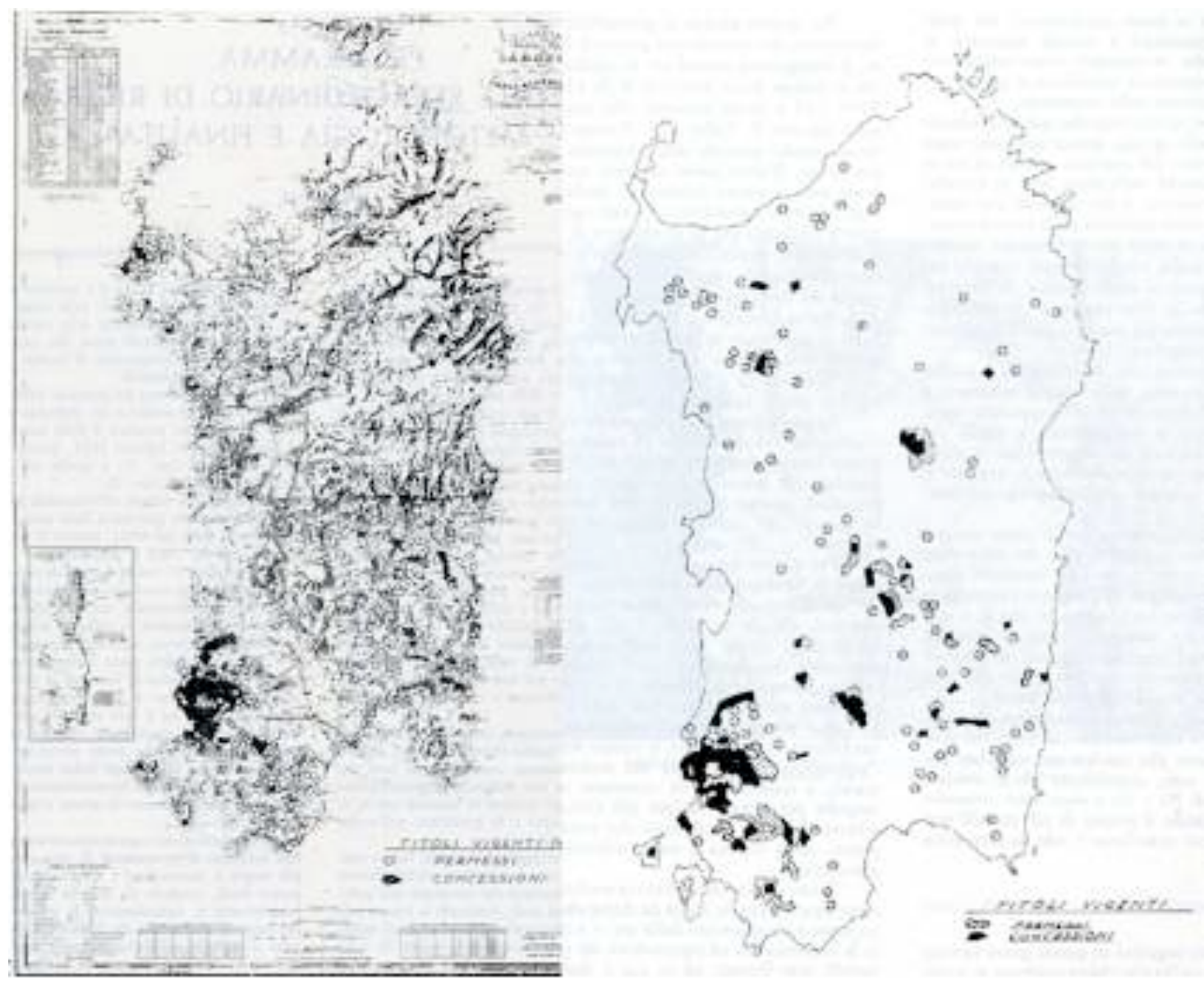

Fonte: Notiziario tecnico-economico EMSa, 1973, pp. 10-11. 
Tabella 1 - Produzione dei concentrati 1951-1971 (tonn)

\begin{tabular}{|c|c|c|c|}
\hline SETTORI & 1951 & 1961 & 1971 \\
\hline Galena & 92,6 & 82,7 & 70,7 \\
\hline blenda & 54,4 & 49 & 40,6 \\
\hline Calamina & 90,4 & 77,6 & 99,5 \\
\hline solfuri misti pb zn & - & - & 100 \\
\hline Pirite & 4,2 & 1,4 & 1,3 \\
\hline Rame & 98,6 & 59,9 & 23,3 \\
\hline Antimonio & 62,2 & 100 & - \\
\hline Manganese & 0,2 & 0,5 & - \\
\hline Arsenico & 100 & 100 & - \\
\hline Ferro & 1,3 & 22,8 & - \\
\hline Alluminio & - & 1,7 & - \\
\hline Baritina & 33,3 & 58,3 & 68,6 \\
\hline Fluorina & 11,9 & 26,4 & 64,4 \\
\hline Argille & 15,3 & 12,7 & 27 \\
\hline Caolino e talco & 21,6 & 25,3 & 34,8 \\
\hline Carbone & 52,9 & 31,7 & 16,2 \\
\hline Acque minerali & 2 & 2,4 & 1,6 \\
\hline TOTALE & $\mathbf{3 2 , 3}$ & 21 & $\mathbf{1 5}, 5$ \\
\hline
\end{tabular}

Fonte: EMSa (a cura di), Ipotesi per un piano minerario regionale, Cagliari, s.e., 1973, tavola 9, p. 52.

Tabella 2 - Addetti all'industria estrattiva: confronto Sardegna-Italia 19511961

\begin{tabular}{|c|c|c|c|c|c|c|}
\hline Ripartizioni & \multicolumn{3}{|c|}{ Censimento 1951 } & \multicolumn{3}{c|}{ Censimento 1961 } \\
\cline { 2 - 6 } & $\begin{array}{c}\text { Addetti } \\
\text { industrie } \\
\text { estrattive }\end{array}$ & $\begin{array}{c}\text { Addetti } \\
\text { industrie }\end{array}$ & $\begin{array}{c}\text { \% sul } \\
\text { totale } \\
\text { occupati } \\
\text { secondo } \\
\text { settore }\end{array}$ & $\begin{array}{c}\text { Addetti } \\
\text { industrie } \\
\text { estrattive }\end{array}$ & $\begin{array}{c}\text { Addetti } \\
\text { industrie }\end{array}$ & $\begin{array}{c}\% \text { sul } \\
\text { totale } \\
\text { occupati } \\
\text { secondo } \\
\text { settore }\end{array}$ \\
\hline
\end{tabular}




\begin{tabular}{|c|c|c|c|c|c|c|}
\hline Sardegna & $24.550,00$ & $68.501,00$ & 35,83 & $13.270,00$ & $69.979,00$ & 18,96 \\
\hline Italia & $118.662,00$ & $4.241 .901,00$ & 2,79 & $101.214,00$ & $5.635 .322,00$ & 1,79 \\
\hline $\begin{array}{c}\text { \% Sardegna } \\
\text { sul totale } \\
\text { occupati } \\
\text { industrie } \\
\text { estrattive } \\
\text { Italia }\end{array}$ & 20,68 & 1,68 & & 13,11 & 1,24 & \\
\hline
\end{tabular}

Fonte: CAMERE DI COMMERCIO, INDUSTRIA E AGRICOLTURA DELLA SARDEGNA (a cura di), op. cit., p. 62.

Tabella 3 - Sardegna, industria mineraria: Occupazione 1951-1979

\begin{tabular}{|c|c|c|c|c|c|}
\hline SETTORI & 1951 & 1961 & 1971 & 1976 & 1979 \\
\hline $\begin{array}{c}\text { Piombo e } \\
\text { zinco }\end{array}$ & $8.126,00$ & $5.040,00$ & $3.100,00$ & $2.494,00$ & $1.846,00$ \\
\hline Rame & 135,00 & 103,00 & 122,00 & 110,00 & 114,00 \\
\hline Antimonio & 275,00 & 87,00 & 24,00 & 13,00 & 13,00 \\
\hline Manganese & 18,00 & 23,00 & 0,00 & 0,00 & 0,00 \\
\hline Arsenico & 115,00 & 41,00 & 0,00 & 0,00 & 0,00 \\
\hline Ferro & 57,00 & 534,00 & 0,00 & 0,00 & 0,00 \\
\hline Alluminio & 0,00 & 125,00 & 0,00 & 0,00 & 0,00 \\
\hline Baritina & 178,00 & 390,00 & 731,00 & 274,00 & 249,00 \\
\hline Fluorina & 90,00 & 440,00 & 313,00 & 561,00 & 521,00 \\
\hline Argille & 133,00 & 108,00 & 66,00 & 100,00 & 101,00 \\
\hline Caolino & 237,00 & 275,00 & 182,00 & 155,00 & 119,00 \\
\hline Carbone & $8.905,00$ & $2.731,00$ & $1.177,00$ & 318,00 & 180,00 \\
\hline Totale & $18.296,00$ & $9.797,00$ & $5.715,00$ & $4.025,00$ & $3.159,00$ \\
\hline
\end{tabular}

Fonte: EMSa, Ipotesi... op.cit., tavola 13 e Notiziario tecnico-economico EMSa, 1-2/1980. 


\section{Indice delle sigle delle Società minerarie}

AMMI Associazione Mineraria Metallurgica Italiana

AMMISarda costola regionale dell'Associazione Mineraria Metallurgica Italiana

EGAM Ente Gestione Attività Minerarie

EMSa Ente Minerario Sardo

ENI Ente Nazionale Idrocarburi

SAMIM Società Azionaria Minerario-Metallurgica

SMCS Società Mineraria Carbonifera Sarda

SOGERSA Sociètà Gestione Risorse Minerarie Sarde 


\section{* L'autore}

Francesca Sanna ha conseguito la Laurea triennale in Storia cum laude presso l'Università degli Studi di Milano nel 2012 con una tesi sull'industria estrattiva sarda del secondo dopoguerra; in seguito si è iscritta alla Laurea Magistrale in Scienze storiche dell'Università di Bologna, dove è stata selezionata per il Corso integrato in Storia e Civiltà comparate in cotutela con l'Université Denis Diderot - Paris 7. Attualmente sta svolgendo a Parigi le ricerche per la sua tesi di Laurea magistrale, incentrata sullo studio dell'organizzazione scientifica del lavoro nel primo dopoguerra.

URL: < http://www.studistorici.com/progett/autori/\#Sanna_Francesca>

\section{Per citare questo articolo:}

SANNA, Francesca, «La miniera e il petrolchimico. Una questione storica nella Sardegna e nell'Italia del secondo dopoguerra», Diacronie. Studi di Storia Contemporanea : Periferie. Cultura, economia, politica, 29/3/2014, URL: < http://www.studistorici.com/2014/3/29/sanna_numero_17/ >

Diacronie Studi di Storia Contemporanea $\beta$ www.diacronie.it

Risorsa digitale indipendente a carattere storiografico. Uscita trimestrale. redazione.diacronie@hotmail.it

Comitato di redazione: Jacopo Bassi - Luca Bufarale - Elisa Grandi - Deborah Paci - Fausto Pietrancosta - Matteo Tomasoni - Luca Zuccolo 